\title{
¿Poder material o poder social? Reflexiones sobre las negociaciones multilaterales y el poder climático de la Argentina (2003-2015) ${ }^{1}$
}

\author{
Material or Social Power? Reflections on the multilateral negoti- \\ ations and the climatic power of Argentina
}

\section{María del Pilar Bueno² y Patricio Yamin Vázquez ${ }^{3}$}

Resumen: La presente contribución aborda la cuestión del poder climático de Argentina en el contexto de las negociaciones multilaterales en la materia, durante las presidencias de Néstor Kirchner y Cristina Fernández (2003-2015). Tal objeto es analizado a partir de dos enfoques teóricos de las Relaciones Internacionales. El primer enfoque propone una perspectiva materialista y racionalista que busca analizar la acumulación de poder del actor estatal y sus implicancias en la posición internacional de Argentina en la Convención Marco de Naciones Unidas sobre Cambio Climático. El segundo enfoque, desde una perspectiva constructivista, propone comprender la construcción del poder climático social en la Argentina desde el proceso de internalización de las identidades y los intereses de diversos actores.

Palabras clave: Poder Climático; Argentina; Política Climática; Liberalismo; Constructivismo

Abstract: This contribution addresses the issue of Argentina's climate power in multilateral negotiations on climate change during the presidencies of Néstor Kirchner and Cristina Fernández (2003-2015). This object is analyzed from two theoretical approaches to International Relations. The first approach proposes a materialist and rationalist perspective that seeks to analyze the accumulation of power of the state and its implications in the international position of Argentina in the United Nations Framework Convention on Climate Change. The second approach, from a constructivist perspective, proposes to understand the construction of the social climatic power in Argentina from the process of internalization of the identities and the interests of diverse actors.

Key words: Climate Power; Argentina; Climate Policy; Liberalism; Constructivism

1 Recibido: 22/05/17. Aceptado: 20/09/17

2 Doctora en Relaciones Internacionales. Investigadora del Consejo Nacional de Investigaciones Científicas y Técnicas de la Argentina (CONICET). Integrante del Comité de Adaptación de la Convención Marco de Naciones Unidas sobre Cambio Climático (CMNUCC). Negociadora del Acuerdo de París y de su implementación. Docente de grado y postgrado en la Universidad Nacional de Rosario (UNR), Universidad Nacional de La Plata (UNLP) y Universidad Nacional de Entre Ríos (UNER). mbueno@conicet.gov.ar

3 Magíster en Relaciones Internacionales (Institut Barcelona d'Estudis Internacionals - IBEI) y Licenciado en Relaciones Internacionales (Universidad del Salvador). Becario doctoral del Consejo Nacional de Investigaciones Científicas y Técnicas de la Argentina (CONICET). Docente de la Universidad de San Andrés (UdeSA).pyaminvazquez@udesa.edu.ar 


\section{Introducción}

El cambio climático se ha convertido en uno de los principales temas de la agenda política global, en tanto sus consecuencias se hacen progresivamente más notorias y los plazos para afrontar el problema se acortan, teniendo en cuenta la información provista por la ciencia climática. En este contexto, la presente contribución aborda la cuestión del poder climático, un concepto polisémico cuyo análisis puede remontarse a distintos enfoques teóricos de las Relaciones Internacionales, con el objeto de contribuir a la comprensión, tanto de las negociaciones internacionales sobre la materia en general, como así también de la posición argentina.

Tal como afirma Creus (2013), el concepto de poder adquiere centralidad en el análisis realista, en sus distintas vertientes. Sin embargo, el cambio climático ha sido analizado en mayor medida desde la impronta liberal institucionalista, especialmente enfocándose en los regímenes internacionales y su interpretación a través del concepto de complejo regimental y complejo regimental transnacional (Yamin, Depledge, 2004; Keohane, Victor, 2010; Abbott, 2012). En menor medida, se evidencian análisis sobre el poder desde la perspectiva constructivista (Wendt, 1992; Haas, 2004; Guzzini, 2005), que priorizan el proceso de construcción social de poder, el cambio climático y su relación con las ideas, creencias e identidades de una sociedad (Rosa, Dietz, 1998; Pettenger, 2007; Bueno, 2013, 2016).

Desde una mirada constructivista, Alexander Wendt (1999), tiende puentes epistemológicos entre el denominado mainstream de las Relaciones Internacionales, compuesto principalmente por los pensamientos liberal y realista, y otras teorías sociales que usualmente se han denominado reflectivistas. Sin embargo, y al mismo tiempo, presenta diferencias ontológicas sustantivas con el racionalismo.

En términos epistemológicos, Wendt no cuestiona la importancia de la distribución y la regulación del poder. Tampoco critica el rol del Estado, que sigue siendo un actor significativo y prioritario de la sociedad internacional ${ }^{4}$, aunque afirma que el Estado liberal está constreñido por actores no estatales y que éstos pueden ser centrales para comenzar un proceso de cambio social. Sin embargo, es el Estado el que, en última instancia, garantiza el cambio sistémico (Wendt, 1999:).

Los Estados están compuestos por actores con intereses, identidades y racionalidades diversas, incluyendo a los tomadores de decisiones, lo cual ya había sido examinado en gran parte por el institucionalismo, y en tal sentido se evidencia un nuevo puente. Sin embargo, el cambio radica en la ontología de la propuesta. Dado que tanto el realismo como el institucionalismo presentan una ontología materialista, por la cual, la base material determina la estructura y la distribución de poder. Tanto el neorrealismo waltziano como el institucionalismo reconocen que las ideas importan pero no ven que el poder y los intereses sean efectos de las ideas (Wendt, 1999). El constructivismo parte de considerar que son las ideas, constitutivas de la estructura social junto con la base material y los intereses, las que otorgan significado a los otros dos elementos. En la medida en la cual el constructivismo valora en mayor proporción el rol de las ideas por sobre la base

4 Afirma Wendt que el Estado es la forma predominante de subjetividad contemporánea en la política mundial y que en tal sentido, deben ser la unidad primaria de análisis para pensar la regulación global de la violencia (Wendt, 1999: 9). 
material, consiente y promueve el cambio social explicado a partir de cómo las estructuras sociales son el efecto de las prácticas sociales.

Es así que el constructivismo puede ser visto en Wendt como un estructuralismo idealista en la medida que, por un lado, considera que las estructuras de la asociación humana están determinadas en forma primaria por las ideas compartidas antes que por fuerzas materiales y, por otro lado, que las identidades e intereses de los actores están constituidas por ideas compartidas y no dadas naturalmente. Por este motivo, la anarquía internacional es una construcción social y no el Estado de naturaleza (Wendt, 1992, 1999).

Con lo cual, el poder, los intereses y las capacidades materiales importan en el pensamiento de Wendt, así como la estructura social que integran. Sin embargo, la estructura alude a las disposiciones de la asociación humana, y por ende, responde a aspectos primeramente culturales más que materiales, y la cultura no es otra cosa que el conocimiento socialmente compartido (Wendt, 1999). Es por esto que la estructura social anárquica es una construcción, así como lo son las culturas hobbesiana, lockeana y kantiana, que se encuentra en profunda relación con condiciones cooperativas y conflictivas de las relaciones sociales.

La valoración de todos los elementos que componen la estructura social y especialmente la noción de "idea" como meta-concepto constructivista, permiten pensar y explicar el cambio social, que en otros aportes teóricos resulta irrelevante o imposible. La estructura social es co-constitutiva del proceso (interacción y aprendizaje) como la relación dinámica existente entre agente y estructura.

Al asumir el cambio, como las modificaciones que se dan en las ideas y creencias de los agentes, se destierra la visión de las Relaciones Internacionales como una estructura insondable y material que trasciende al individuo y a la sociedad. Esto facilita analizarlas como un entramado, una realidad que la sociedad construye en virtud de un proceso intersubjetivo (Bueno, 2010).

Por lo expuesto, proponemos analizar el poder climático de un actor estatal concreto, como es Argentina, en el contexto de las negociaciones multilaterales de cambio climático durante las presidencias de Néstor Kirchner y Cristina Fernández (2003-2015). La primera parte de la contribución, se adentra en el análisis del objeto desde una perspectiva materialista y racionalista que busca examinar la acumulación de poder del actor estatal y sus implicancias en la posición internacional de Argentina en la Convención Marco de Naciones Unidas sobre Cambio Climático.

En la segunda parte y desde una perspectiva constructivista, buscamos comprender la construcción del poder climático social en la Argentina desde el proceso de internalización de las identidades e intereses sociales.

Cabe mencionar que esta contribución se concentra en la Política Exterior argentina comprendida como política pública y, en tal sentido, esto no implica analizar al Estado como actor racional unificado, sino comprender que dicha política es la proyección internacional de la sociedad argentina en constante relación e interactuando con aspectos domésticos. Se debe aclarar, no obstante, que la distinción nacional-internacional de modo rígido tiene tan sólo una lógica analítica ${ }^{5}$. 
A partir de las diferencias ontológicas entre los dos enfoques analíticos seleccionados, cada aproximación delimita un aporte propio al debate sobre el poder climático de Argentina en el período estudiado. El sentido de analizar un mismo objeto desde dos ópticas teóricas distintas proviene de considerar que tales teorías constituyen herramientas analíticas que imprimen un sello inevitable en la realidad observada. De este modo, una mirada material favorece un análisis sobre búsqueda, acumulación y preservación del poder, siendo que uno de carácter constructivista considera que dicha condición material no es determinante, sino sólo un aspecto de la estructura social. Mientras el primer enfoque promueve un análisis de consecuencias para comprender una realidad material que toma como dada, así como de estrategias relativas al poder negociador de la Argentina, el segundo, procura desentrañar las causas, en tanto creencias e identidades que constituyen el proceso de construcción social del poder, habilitando cambios en las condiciones de su tratamiento.

El diseño metodológico es de tipo cualitativo, empleando técnicas de recolección de datos provenientes de fuentes primarias (documentos oficiales de Argentina y de la CMNUCC) y secundarias (artículos publicados en revistas indexadas, así como libros y capítulos de libros que constituyen el aporte teórico principal), mientras que las técnicas de análisis de información surgen del análisis documental, así como cualitativo y estadístico.

El recorte temporal se basa en aspectos domésticos e internacionales. Por un lado, se observa una continuidad política en los tres mandatos comprendidos en el período estipulado, así como también se presenta un cambio de signo político en diciembre de 2015, con la llegada a la Presidencia de la Coalición Cambiemos y la asunción de Mauricio Macri. Del mismo modo, el período comprendido abarca algunos de los momentos más relevantes del período posterior a la entrada en vigor del Protocolo de Kioto, desembocando en la Conferencia de París de diciembre de 2015, que se constituye como el hito reciente más importante de las negociaciones.

\section{Poder material climático}

Para analizar el poder dentro de las negociaciones sobre cambio climático desde una perspectiva materialista, partimos de una conceptualización que observa dos dimensiones del mismo. Por un lado, abordamos el poder en tanto capacidades materiales, en una definición asociada a los enfoques clásicos de la disciplina; mientras que, por el otro, buscamos comprender el poder de negociación que los Estados presentan como consecuencia del ámbito específico en el que se encuentren y las reglas de juego asociadas al mismo (McKibben, 2015). Ésta última se enmarca en la reflexión del neoliberalismo institucional de las Relaciones Internacionales, así como también en la literatura sobre cooperación y teoría de los juegos.

La concepción realista sobre el poder resulta ineludible en cualquier trabajo que aborde su estudio desde una perspectiva material. En palabras de Morgenthau (1986: 20), el poder refiere a "cualquier cosa que establezca y mantenga el control del hombre sobre el hombre", o dicho de otro modo, "el poder del hombre sobre las mentes y las acciones de otros hombres". Más allá de los diferentes matices entre autores, el consenso realista marca la preponderancia de la geografía, el poder militar y los recursos económicos a la hora de medir el poder (Bially Mattern, 2010). Kenneth Waltz (1988: 193-194) afirma que el poder de los Estados "depende del modo en que se sitúen en 
todos los aspectos siguientes: dimensión de población y de territorio, recursos, capacidad económica, fuerza militar, estabilidad, y competencias políticas"; John Mearsheimer (2001: 131-132) señala que el poder se basa en las "capacidades materiales particulares que un Estado posee" y que hacen referencia, principalmente, a tres cuestiones: población, riqueza y fuerza militar.

Un punto esencial en esta forma de medir el poder lo constituye el hecho de que la distribución de las capacidades es indiferente del área temática de aplicación: Waltz (1988: 193) afirma que las capacidades "no pueden ser sectorizadas y evaluadas por separado". En su caracterización del realismo, Keohane y Nye (1988: 48) observan que "los Estados fuertes asegurarían la congruencia entre la estructura global del poder militar y económico y el patrón de resultados en cualquier área de cuestiones".

No obstante, el realismo encuentra limitaciones en el análisis. La eficacia de la fuerza militar en todas las áreas de cuestiones descansa sobre el presupuesto de que la seguridad de los Estados está amenazada. Sin embargo, observamos que en tanto los Estados dejan de ver amenazada su supervivencia, la utilización de la fuerza se torna más costosa y menos eficiente de aplicar en otras cuestiones. Por el contrario, cada área de cuestiones presenta sus propios recursos de poder, que no necesariamente coinciden con el poder económico y, especialmente, con el poder militar: “así, mientras la utilidad de la fuerza disminuye (...), la distribución de poder dentro de cada cuestión se tornará más importante" (Keohane y Nye, 1988: 49).

Esto plantea el interrogante de definir los elementos para identificar la distribución de poder dentro del área de cambio climático. El indicador más utilizado para realizar esta medición es el porcentaje sobre las emisiones globales del que es responsable cada Estado, asentado sobre la correlación que existe entre el nivel de emisiones y la capacidad de afectar los resultados de las negociaciones internacionales en la materia (Paterson 2003: 98). Siguiendo esta línea, Viola, Franchini y Lemos Ribeiro (2012) plantean que para identificar las potencias climáticas es necesario combinar las dos dimensiones tradicionales del poder, es decir la militar y la económica, con la dimensión climática del poder, sosteniendo que existe una fuerte interrelación entre los tres elementos (Franchini, 2011). En esta última, los autores incluyen el volumen y la trayectoria de las emisiones de los gases de efecto invernadero, el capital humano y tecnológico disponible para la transformación hacia una economía de bajo carbono, y la relación entre los recursos y la cultura energética, así como también se aclara que no se excluyen factores no materiales, tales como la influencia o el prestigio. No obstante, los factores materiales, tanto los generales como los específicos del área, son los determinantes a la hora de realizar el análisis y la clasificación de los países en las negociaciones climáticas.

Del mismo modo, debemos prestar atención a los espacios institucionales en que se insertan los actores y como éstos influyen sobre sus estructuras de incentivos para la acción. Uno de los principales supuestos de la escuela liberal es que la política internacional se encuentra institucionalizada y que las instituciones no son epifenoménicas como afirma gran parte del realismo, sino que por el contrario, importan en tanto afectan los costos y beneficios con los que se enfrentan los Estados (Keohane, 1993). En este sentido, se resalta la faceta del poder que está constituida por el posicionamiento institucional de los actores (Bially Mattern, 2010: 693).

En este punto, retomamos la conceptualización de McKibben (2015: 17-19) sobre el poder de negociación, que se deriva de las reglas que establece un determinado ámbito institucional. Esta segunda clase de poder se basa principalmente en los costos que el 
Estado observaría si no se alcanzara un acuerdo cooperativo. En el caso de que el Estado tenga como mejor alternativa a no alcanzar un acuerdo una opción demasiado costosa, será más proclive a ofrecer concesiones $\mathrm{y}$, en consecuencia, tendrá menos poder para determinar el resultado de las negociaciones finales. En las negociaciones ambientales la vulnerabilidad frente al problema que se esté buscando afrontar es la clave para comprender los costos que los Estados enfrentan en el caso de no alcanzar una solución multilateral (Rong, 2010; McKibben, 2015).

Cabe destacar que el poder material y el poder de negociación no necesariamente coinciden. Por el contrario, en sus diferencias aparecen las estrategias de los Estados en una negociación. En el caso de tener un bajo poder de negociación pero un alto poder material, el Estado puede hacer valer este último para modificar las reglas y, consecuentemente, mejorar su posición institucional. Por el contrario, si tiene ambos niveles de poder con valores bajos, será proclive a cumplir con las reglas establecidas.

Es necesario observar que el hecho de que un Estado cumpla con las reglas (ya sea por conveniencia o por falta de poder material para modificarlas), no aporta información sobre qué tipo de postura tomará dentro de la negociación. En este sentido, los Estados pueden adoptar estrategias cooperativas o conflictivas (McKibben, 2015). Esto se encuentra determinado por tres factores. En primer lugar, el costo de la alternativa a no acordar: los Estados con altos costos tienen menos margen para confrontar que aquellos que tienen bajos costos. En segundo, la estructura y valoración de temas que se encuentran incluidos en una negociación. Si los Estados otorgan el mismo valor a los mismos temas, es más probable que adopten estrategias confrontativas que si valoran de forma diferente los distintos puntos de la negociación. Esto es consecuencia de que, frente a diversas escalas de valores de los Estados, una negociación que involucre concesiones mutuas es más sencilla.

En tercer y último lugar, debemos tener en cuenta si los Estados se focalizan en las ganancias absolutas o relativas. Mientras que un foco en las primeras hace más fácil llegar a un acuerdo, el foco en las segundas lo hace más complejo en tanto la preocupación por los Estados no está solo enfocada en las ganancias propias, sino en como éstas se comparan con las de los demás. Este segundo caso se observa particularmente en negociaciones relacionadas con la seguridad o que afecten la competitividad de los Estados, en tanto ambas áreas no dependen de las capacidades absolutas, sino de su comparación con los recursos de los demás (McKibben, 2015).

\section{El poder climático material y de negociación de Argentina}

En base al marco teórico racional-materialista precedente, afirmamos como hipótesis que "Argentina ha tenido una posición de poder medio en las negociaciones climáticas y que ha buscado conservar su poder mediante estrategias defensivas de confrontación, sin que esto signifique un no cumplimiento de las reglas o una apuesta por fuera de las instituciones establecidas, en tanto presenta un bajo poder de negociación entendido como capacidad para extraer concesiones de las otras partes". De este modo, abordamos la misma a partir de dos puntos centrales: su posicionamiento material como potencia media y vulnerable, y su estrategia defensiva. 


\subsection{Una potencia media con vulnerabilidad relativa}

En este punto se resalta que Argentina aparece como una potencia media, con una influencia limitada en las negociaciones. Esto se desprende de una dotación de poder duro modesta, una economía mediana y una participación en las emisiones marginal, que hace que no sea un actor de primera necesidad para alcanzar un acuerdo. Cabe resaltar que esta clasificación coincide con la realizada por Viola, Franchini y Lemos Ribeiro en donde ubican al país como una potencia media o incluso "medio baja" (Franchini, 2011: 185; Viola, Franchini, Lemos Ribeiro, 2012).

Tal como se especificó con anterioridad, los puntos esenciales para evaluar los recursos materiales son las capacidades generales, por un lado, y las climáticas, por el otro. En cuanto a su poder sistémico, podemos recurrir al Índice de Capacidades Nacionales, elaborado por "The Correlates of War Project", y que incluye la producción metalúrgica y siderúrgica, el gasto y personal militar, el consumo de energía, la población total y la población urbana (NMC v5.0) ${ }^{6}$. Siguiendo el indicador del año 2012, Argentina se ubica en el cuarto lugar en la región, por detrás de Brasil, México y Colombia; y en la posición número 32 en términos globales. En términos específicos de gasto militar, no sólo se observa que el mismo es limitado, sino que además disminuye durante gran parte del período. De acuerdo a los datos de los "World Development Indicators"del Banco Mundial, el gasto militar como porcentaje del Producto Bruto Interno (PIB) disminuyó anualmente y de forma progresiva entre 2003 y 2008. A partir de dicho año se observa un aumento, aunque siempre por debajo del valor del primer año del período. De hecho, el país se ubicó consistentemente por debajo de la media latinoamericana durante todo el período ${ }^{7}$. Su relevancia en este rubro es claramente baja y no se ha buscado impulsar este factor de poder.

En términos económicos, Argentina aparece como un país de relevancia media. De acuerdo a las diferentes medidas del Fondo Monetario Internacional y del Banco Mundial, el país figura entre la posición vigésima y trigésima en la tabla de economías más grandes del planeta. Si bien su participación en el Producto Bruto mundial se encuentra por debajo del $1 \%$ y es por lo tanto limitada, esto está condicionado por el notable peso de las dos primeras economías mundiales, Estados Unidos y China. Cabe destacar que el hecho de ser miembro del G-20 nos permite también afirmar su relevancia media en este rubro.

En cuanto a la dimensión climática del poder material, su relevancia también aparece como media. Esto se deduce principalmente del hecho que el país influye de modo marginal en las emisiones globales, pero se encuentra de forma consistente entre los 30 mayores emisores. De acuerdo a las bases de datos del World Resources Institute, entre 1990 y 2012 Argentina nunca superó el 1\% de las emisiones globales y las proyecciones esperadas no auguran un aumento relevante de las mismas ${ }^{8}$.

6 National Material Capabilities (NMC) v 5.0. Disponible en http://www.correlatesofwar.org/datasets/national-material-capabilities Último acceso: febrero de 2017.

7 Tomado de The Quality of Government Standard Dataset, version Jan17. Disponible en http://www.qog.pol.gu.se doi:10.18157/QoGStdJan17 Último acceso: febrero de 2017.

8 World Resources Institute, CAIT Database. Disponible en http://cait.wri.org/. Último acceso: enero de 2017. 
Del mismo modo, la vulnerabilidad aparece como otro de los factores relevantes para comprender el poder del actor en la negociación. Como punto de partida, es necesario observar que la vulnerabilidad puede analizarse tanto en términos absolutos como relativos a otros Estados, en tanto esto permite obtener la medida de poder de negociación y la capacidad de extraer concesiones de las otras partes. En los países en desarrollo la vulnerabilidad suele estar ligada a tres factores: el impacto sobre la agricultura, el aumento del nivel del mar, y las pérdidas asociadas a desastres naturales vinculados al cambio climático (Rong, 2010). En el caso particular de Argentina, el primero de los tres puntos reviste particular importancia.

La Tercera Comunicación Nacional alerta sobre la existencia de varios riesgos que elevan la vulnerabilidad del sector: el aumento de eventos climáticos extremos; la mayor variabilidad pluvial; la expansión de la frontera agrícola y la degradación de los suelos (Secretaría de Ambiente y Desarrollo Sustentable, 2015: 130-131). Asimismo, el problema de la disponibilidad del agua amenazaría particularmente a las regiones de Cuyo y Norte. Del mismo modo, se menciona también la mayor cantidad de inundaciones por el incremento de las precipitaciones o el aumento de la demanda eléctrica por las temperaturas más elevadas ${ }^{9}$.

Por su parte, las pérdidas asociadas a desastres naturales relacionados con el cambio climático colocan al país en una posición de vulnerabilidad, si bien no extrema, considerable. Diversos índices apuntan a medir los potenciales peligros ambientales que enfrentan los países. Entre ellos, el "ND-GAIN Country Index", de la Universidad de Notre Dame, contempla la vulnerabilidad al cambio climático en combinación con su capacidad de hacer frente a la misma ${ }^{10}$. Tomando en consideración la menor vulnerabilidad y la mejor posibilidad de adaptación, Argentina aparece en la posición 85 sobre 181 países. Es necesario destacar que, dentro de las vulnerabilidades, los puntos más susceptibles de cambios son la agricultura y la dependencia del agua por habitante. El "Global Climate Risk Index"elaborado por la organización German Watch, por su parte, toma en consideración las pérdidas en términos de vidas humanas y recursos económicos. En el mismo, Argentina se ubicó en el año 2015 en la posición 63 de países con mayores pérdidas sobre un total de 180 y en la posición 92 en todo el período 1996-2015 (Kreft, Eckstein y Melchior, 2017). Cabe destacar que en el año 2013, el país estuvo entre los 10 países del mundo con mayores costos asociados a este tipo de fenómenos.

Por último, la vulnerabilidad ambiental del país en términos generales aparece en un nivel medio. De acuerdo al Índice de Vulnerabilidad Ambiental elaborado por la Comisión de Geociencia Aplicada del Pacífico Sur (SOPAC) y el Programa de Naciones Unidas para el Medio Ambiente (PNUMA), Argentina se ubica como un país "vulnerable", por debajo de los países "muy vulnerables" y "extremadamente vulnerables"11. Además del cambio climático, este índice cubre dimensiones como biodiversidad, agua, agricultura y pesca, salud humana, desertificación, y exposición a desastres naturales.

9 Esta información se condice con los informes del Panel Intergubernamental de Expertos sobre Cambio Climático (AR5, 2014).

10 ND-GAIN Country Index. Disponible en http://index.gain.org/. Último acceso: marzo de 2017.

11 Environmental Vulnerability Index. Disponible en http://www.vulnerabilityindex.net. Último acceso: septiembre de 2017. 
Por lo tanto, Argentina presenta una situación de vulnerabilidad al cambio climático que, si bien es inferior a otros Estados, es relevante. Esto es producto principalmente de las potenciales consecuencias sobre las actividades agropecuarias en términos económicos y sociales que van asociadas a los cambios en la producción. En esta línea, Franchini señala que Argentina presenta una "vulnerabilidad difusa": si bien es alta por ser un país en desarrollo y por su perfil productivo, no es extrema ni inmediata (Franchini, 2011).

Desde una mirada institucionalista, esto conlleva que Argentina necesita un acuerdo multilateral para enfrentar las consecuencias del cambio climático. Asimismo, el hecho de ser un país con poder material medio no le permite plantear una estrategia de cambios de regla de juego, sino que debe atenerse a los márgenes de la negociación establecidos por las grandes potencias climáticas. Con lo cual, la cooperación climática multilateral puede ofrecerle mayores márgenes de incrementar su poder negociador que una estrategia bilateral o minilateral.

\subsection{La estrategia defensiva}

Los determinantes de la elección del tipo de estrategia incluyen tres factores: los costos asociados a no tener un acuerdo, la valoración de los temas, y el tipo de ganancias en que se hace foco. El primero de ellos fue abordado en el punto anterior. Teniendo un nivel medio de vulnerabilidad climática, Argentina necesita de un acuerdo climático, pero no se encuentra en una posición de necesidad extrema. Esto permite que aún frente a la necesidad del acuerdo, la diplomacia climática argentina pueda plantear una posición de confrontación limitada o, dicho de otro modo, defensiva dentro del esquema multilateral. Tal es el caso que se sostiene en el presente trabajo sobre la Política Exterior climática durante los gobiernos de Kirchner y Fernández.

Abordamos entonces las causas de la estrategia defensiva, a partir de las posiciones sobre los principales temas de negociación del país, y del foco en las ganancias relativas.

Entre los principales lineamientos temáticos, cabe destacar la creciente oposición a los mecanismos de flexibilización o mercado; una dura defensa del principio de Responsabilidades Comunes pero Diferenciadas (CBDR, de acuerdo a su sigla en inglés); el condicionamiento de los compromisos de mitigación a la llegada de financiamiento externo; y especialmente un rechazo de los compromisos sectoriales que involucren a la agricultura, siendo este tema el de mayor sensibilidad (Franchini, 2011; Bueno, 2012). Estas posiciones chocan con las sostenidas por una parte importante de los países desarrollados, principalmente aquellos en posiciones menos reformistas y más proclives a establecer menores diferencias entre los países desarrollados y aquellos en vías de desarrollo.

Una de las decisiones principales en la estrategia defensiva de Argentina ha sido la incorporación del país al grupo de negociación Like Minded Developing Countries (LMDC), ubicado dentro de las posiciones más proclives a defender la vigencia de la Convención, sus principios y estructura de anexos. La incorporación y canalización de las acciones políticas a través de las coaliciones funciona, en este marco teórico, como una estrategia que busca mejorar el poder de negociación inicial o, al menos, mantener una línea defensiva sobre los elementos más valorados por el país en la negociación. Esto es especialmente relevante siendo un actor que carece de poder material suficiente para conseguir por sí mismo sus objetivos en dicho punto, de modo que busca la canalización 
de su interés a través de una coalición de Estados que le permita mantener la estrategia defensiva.

Del mismo modo, el foco sobre las ganancias relativas también cumple un rol fundamental para la adopción de la estrategia defensiva. En este sentido, existen factores estructurales y domésticos que explican esta posición. Entre los primeros, afirmamos que las negociaciones sobre cambio climático están íntimamente relacionadas con la competitividad económica, en tanto las decisiones adoptadas en términos de mitigación afectan directamente al modo de producción y las estructuras de costos. Esto genera incentivos particulares para que los Estados se preocupen por las ganancias relativas (McKibben, 2015).

Sin embargo, no todos los Estados similares a Argentina adoptaron posiciones defensivas en las negociaciones. De este modo, surge la necesidad de abordar factores no externos, es decir del nivel del actor. Para ello partimos del supuesto de que las preferencias de los Estados se moldean también a partir de la interacción de actores domésticos que se encuentran en constante interrelación con el entorno, en un contexto de globalización e interdependencia global. En este sentido, la globalización aparece como la fuente principal que moldea las preferencias internacionales de los actores domésticos, afectando sus estructuras de costos y beneficios y, en consecuencia, los incentivos para buscar aumentar o disminuir la interdependencia (Moravcsik, 2008).

En esta línea, Solingen (1998; 2001) afirma que la "gran estrategia" de los Estados es una consecuencia de los diferentes tipos de coaliciones internas que pueden conformarse. Las mismas se definen principalmente en cómo se posicionan frente a la "internacionalización" de la economía, como consecuencia del impacto que la globalización ha tenido, en términos de costos y beneficios, oportunidades y amenazas, para cada uno de ellos. Es esperable, entonces, que aquellos sectores competitivos internacionalmente, orientados a la exportación o a los mercados financieros internacionales, sean más favorables a un proceso de internacionalización liberal, mientras que aquellos sectores no competitivos vean una amenaza creciente en aquel y adopten posiciones defensivas. En consecuencia, los actores domésticos tienden a agruparse en dos coaliciones ideales ubicados en extremos opuestos de un continuo: coaliciones internacionalistas-liberales, por un lado; y coaliciones nacionalistas-proteccionistas, por el otro. Las primeras buscan disminuir las barreras y aumentar el comercio, atraer inversión extranjera y reducir el peso del gasto público, así como también son favorables a incorporarse a las instituciones internacionales que favorezcan la globalización. Por el contrario, las coaliciones nacionalistas-proteccionistas buscan proteger los sectores vulnerables de sus economías, se focalizan en el mercado interno y son proclives a un mayor gasto público, siendo más reticentes a la internalización de su economía y sus políticas (Solingen, 2001).

Los gobiernos de Néstor Kirchner y Cristina Fernández se enmarcaron dentro de lo que se dio en llamar el "giro a la izquierda" o "nueva izquierda" en América Latina, identificado con el segundo tipo (Levitsky y Roberts, 2011). Esto puede ser observado a partir de indicadores como el Índice de Libertad Económica, cuyas dimensiones son: "estado de derecho", incluyendo derecho sobre la propiedad; "gobierno limitado"; "eficiencia regulatoria", entendida como libertad de negocios, de trabajo y monetaria; y "mercados

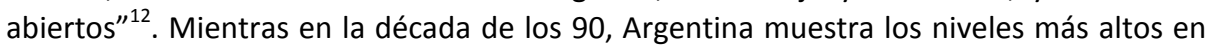


América del Sur junto con Chile, a partir del 2003 se observa una fuerte caída que ubica al país en los puntajes más bajos del índice.

La pertenencia a cierto tipo de coalición se evidencia también en la política exterior. En la base de datos de Merke y Reynoso (2016) sobre dimensiones de política exterior, los gobiernos de Néstor Kirchner y Cristina Fernández se encuentran ubicados dentro de los gobiernos con orientación ideológica pro-Sur, una economía proteccionista y una posición de autonomía frente a los Estados Unidos. De hecho, los tres períodos presidenciales se ubicaron por debajo de la media regional. Se observa además una correlación positiva entre el paso del tiempo y la profundización de estos perfiles, en cuanto los números entre una presidencia y la siguiente, o bien se mantienen, o bien aumentan.

De este modo, la coalición de gobierno doméstica de Néstor Kirchner y Cristina Fernández es propensa a adoptar medidas defensivas frente a las potencias globales y a los países desarrollados, con una posición donde la brecha Norte - Sur es un concepto clave en la planificación política. En este punto, comprendemos la defensa férrea del principio de CBDR, la búsqueda de financiamiento para la adaptación y el intento por proteger los sectores más expuestos de la economía doméstica. En esta etapa las dinámicas internas son las preponderantes para entender la adopción de las estrategias (Bueno, 2015).

\section{Poder climático social}

En este apartado nos proponemos debatir la cuestión del poder climático de Argentina a partir de los aportes del constructivismo de las Relaciones Internacionales.

Hemos planteado que una de las principales críticas del constructivismo al racionalismo alude al efecto directo de las capacidades materiales en los resultados; y a la reificación de la anarquía internacional como una característica ineludible de la estructura del Sistema, desembocando y justificando una visión antropogénica negativa y, por ende, una mirada defensiva de las Relaciones Internacionales. Asimismo, también se critica la lógica exógena de los intereses; la preponderancia del agente o de la estructura dependiendo del enfoque al cual se haga referencia; y la supuesta neutralidad valorativa de las ciencias y del positivismo (Hurd, 2008).

De este modo, el constructivismo abre un debate epistemológico de las Relaciones Internacionales basado en lo que Stefano Guzzini (2005) denomina la desnaturalización del status quo promovido por el mainstream o su inevitabilidad, especialmente en aquellos aspectos que tanto los realistas como los liberales tomaron como dados. De hecho, la acción de definir conceptos constituye para el constructivismo una instancia por la cual se reduce la abstracción y la concepción analítica. Además, toda definición debe enmarcarse en un contexto teórico e histórico, lo que lleva a la afirmación de que no existen conceptos neutrales o avalorativos, así como el análisis conceptual del poder forma parte de la construcción social como ejercicio del poder mismo. Definir al poder es una intervención política (Guzzini, 2005).

Comprendemos aquí al poder como un proceso intersubjetivo. La construcción social del poder es analizada como un proceso de institucionalización de ideas, creencias e identidades, en el entendimiento de que dicha institucionalización alude a la internalización de la sociedad de identidades e intereses. De este modo, se observa que "el proceso de construcción social del poder apunta a la dinámica por la cual los actores, compartiendo creencias y expectativas acerca de los fenómenos sociales, modelan sus intereses 
e identidades compartidos generando un poder que les es propio y único y que trasciende la noción de poder estatal de tipo material" (Bueno, 2012: 53-54).

En tal sentido, proponemos el concepto de poder climático social como el proceso de construcción social de identidades e intereses sobre el cambio climático y sus efectos, desplegados, entre otros aspectos, en políticas climáticas en su dimensión interna y externa. El poder climático social no se concentra en la acumulación de poder, no hace hincapié en qué actores detentan más o menos poder climático, en tanto esta mirada continuaría perpetrando el poder explicativo de la base material de la estructura social.

El poder climático social tampoco es lo mismo que hablar de potencias climáticas o potencias emisoras. Tal como fue analizado anteriormente, otros enfoques realizan un análisis de este punto basado en dimensiones tradicionales y materiales del poder como las militares y económicas. La consecuencia más directa de este modo de pensamiento es que al analizar el poder, su distribución y potencial incremento, se perpetúa el status quo, en tanto se toma al poder como dado. Esto, a su vez, no compromete la "neutralidad de la ciencia", perdiendo así el potencial de la política climática para generar cambios sociales y perpetuando el actual sistema socio-económico y político que causa el cambio climático (Navarrete, 2010).

Desde una mirada material, Argentina se identifica como una potencia climática media, aunque su disposición a actuar no haya sido consecuente, lo cual se evidencia con el concepto de "compromiso climático" donde dicho país se posiciona como una potencia media conservadora (Viola, Franchini y Ribeiro, 2012: 26). Con lo cual, una potencia media climática sólo necesita incrementar su compromiso climático de modo de asumir un papel reformista acorde con el problema climático y la crisis civilizatoria, dejando de resistir las transformaciones necesarias para estabilizar el sistema climático.

De este modo, en ningún punto se cuestiona cómo se produce el cambio climático; cómo sus efectos se distribuyen de modo inequitativo al interior de las fronteras de los Estados; cómo se vinculan los dilemas del desarrollo y la pobreza con el cambio climático y sus efectos adversos; cómo las nuevas potencias climáticas reproducen las mismas pautas de desarrollo que sus antecesores en el dilema del modelo de desarrollo que nos lleva a prácticas insustentables que no se resuelven solamente con una transición energética hacia sociedades hipocarbónicas. En tal sentido, estos enfoques no apuntan a desentrañar las causas, las identidades e intereses detrás de las acciones de los actores y, por ende, no promueven el cambio social, en tanto toman la estructura y distribución de poder como dada, perpetuando una mirada del cambio climático concentrada en quién paga la factura.

La entrada en vigor del Acuerdo de París constituye un punto de llegada en el proceso de negociaciones multilaterales de cambio climático en la CMNUCC. Asimismo, implica un cambio concentrado en la distribución de esfuerzos, es decir, quién debe hacer más y por qué. Cabe recordar que el principio de responsabilidades comunes pero diferenciadas ha sido el ordenador de la diferenciación entre países desarrollados y en desarrollo desde la firma de la CMNUCC. Si bien el Acuerdo de París lo incluye e implementa, su interpretación varía con la transición del concepto de compromiso presente en la Convención y aplicable, especialmente a los países desarrollados, al de contribución que se ajusta a todas las Partes.

Bajo este principio, los Estados se han organizado en grupos en las negociaciones en la CMNUCC con distintas interpretaciones de las responsabilidades, que en su naturaleza contradictoria, podían explicar por qué no fue posible llegar a un nuevo acuerdo 
climático hasta 2015. El constructivismo nos permite trascender el debate sobre cómo el nuevo sistema de contribuciones nacionales puede o no incrementar la eficiencia del régimen climático y el cumplimiento de su objetivo, para analizar cuáles son las creencias e intereses climáticos de los actores y cómo influyen en la construcción de políticas públicas climáticas nacionales e internacionales.

Teniendo esto en cuenta, afirmamos a manera de hipótesis que "la construcción del poder climático social en la Argentina deriva de la baja demanda social climática y de la escasa internalización societaria de las causas y consecuencias del cambio climático como cambio sistémico. En tal sentido, la política climática del período analizado, especialmente en su fase externa, se caracterizó por una concentración en temas de carácter especialmente defensivo en la medida que que se interpretó al cambio climático y a la negociación del Acuerdo de París como una posible amenaza a las políticas de desarrollo".

\section{La construcción de poder climático social en la Argentina}

Las políticas doméstica y externa, en este caso climáticas, se encuentran en un proceso dinámico de co-construcción. En tal sentido, identificamos seis aspectos -no exhaustivos ni inmóviles- de la vinculación entre dichas políticas, cuya dinámica se ve fortalecida en el período bajo análisis.

La primera cuestión a tener en cuenta es que la inserción y el desarrollo del tópico en la agenda política han sido promovidos más por actores externos que internos (Estrada Oyuela, 2007; Bueno, 2010). La inclusión de las temáticas ambientales, en general, no sólo en la agenda política argentina, sino también en la agenda internacional se produce a partir de la confluencia de aspectos multidimensionales en la década del sesenta, que derivan en la celebración de la primera Conferencia de Naciones Unidas sobre Medio Ambiente Humano, en Estocolmo en 1972 (Bueno, 2010) ${ }^{13}$. En el caso del cambio climático es aún más patente a partir de fines de la década del ochenta y luego de la suscripción de la CMNUCC en la Cumbre de la Tierra, en Río de Janeiro en 1992.

El segundo aspecto consiste que, a pesar de haber transcurrido más de cuatro décadas de estos episodios, y tal como señalan Gutiérrez e Isuani (2014), el ambientalismo social -organizaciones y movimientos sociales con reivindicaciones ambientales- y la creación de agencias estatales ambientales no tuvieron mayor impacto hasta el nuevo milenio, donde emergió un ambientalismo social ligado a la generación de conflictos ambientales. Si bien se puede hablar de una agenda pública ambiental y de su encuentro con la agenda gubernamental en tiempos más recientes, el cambio climático continúa cautivo de los vaivenes internacionales. En términos constructivistas podríamos afirmar que este encuentro de agendas constituye parte del proceso de institucionalización como internalización de ideas. Sin embargo, este proceso es gradual, lo que significa que la interpretación sobre las causas, consecuencias e incluso, las prioridades de la acción

13 Estos hechos incluyen investigaciones científicas sobre el daño ambiental en la salud humana, animal y vegetal; informes de organizaciones como el Club de Roma sobre "Los límites al crecimiento"; hambrunas y derrames de petróleo especialmente en países en desarrollo; la generación de partidos verdes en Europa y el incremento de los movimientos ambientales en Estados Unidos, entre otros. Además, y como señalan Gutiérrez e Isuani (2014), el ambientalismo social y estatal en la Argentina se debe entender a través de la influencia de aspectos ideológicos y financieros internacionales. 
política climática, no constituyen aún una identidad. Por otra parte, la identidad por sí misma no moviliza a la acción, sino que requiere del interés que le otorga la fuerza motivacional. Sin embargo, los intereses presuponen identidades porque un actor difícilmente puede saber qué quiere sin saber quién es.

El hecho de que el cambio climático no constituya una prioridad de la acción política, se vincula con el tercer aspecto que es la baja demanda climática social. Un modo de evidenciar esta afirmación es el contenido que arrojan las encuestas de opinión.

En 2014, la Fundación Vida Silvestre ${ }^{14}$ presentó los resultados de una encuesta ambiental realizada por la consultora Poliarquía; y en 2015 se realizó en 75 países una encuesta climática en vísperas a la COP 21 organizada por la World Wide Views Alliance ${ }^{15}$, teniendo a la Red Argentina de Municipios frente al cambio climático como referente local. En el primer caso, se evidencia que dos tercios de los entrevistados se muestran interesados o muy interesados en temas ambientales. En el segundo, un 95\% de los encuestados dicen estar muy preocupados por el cambio climático. No obstante, cuando se cruzan los datos con otras encuestas sobre los principales temas de preocupación de los argentinos en el mismo período, los temas ambientales no se encuentran priorizados. En la Encuesta "Mi Mundo" organizada por las Naciones Unidas ${ }^{16}$, referida a las prioridades para mejorar la calidad de vida y promover el desarrollo sustentable, el primer tema es la educación, seguido por gobiernos honestos y responsables y la alimentación. En cuarto lugar se ubica el acceso al agua potable y en noveno lugar, el cambio climático.

En cuanto a las encuestas de opinión general en 2015, previo a las elecciones nacionales, la consultora Hugo Haime \& Asociados ${ }^{17}$ establecía que los temas que más preocupaban a los argentinos eran la inseguridad (47\%), la corrupción (25\%), la inflación y la desocupación (22\%). Mientras que Management \& $\mathrm{Fit}^{18}$ identificaba a la inseguridad $(80,9 \%)$, a la inflación $(62,9 \%)$ y al desempleo $(52,0 \%)$ como prioridades.

Según el Latinobarómetro, durante 2015 los principales problemas identificados por los argentinos fueron la delincuencia y la seguridad pública (35\%), los problemas políticos $(13.4 \%)$, la desocupación $(12,5 \%)$, la economía $(8,4 \%)$ y la inflación $(8.3 \%)$. El calentamiento global no figura en la lista, a diferencia de otros países de la región como Brasil y Chile donde el tema figura en un $0,2 \%{ }^{19}$.

Las encuestas de opinión ambiental se enfrentan a diversos dilemas, entre ellos "lo políticamente correcto". Esto explicaría, al menos en parte, por qué un ciudadano puede decir que se encuentra muy preocupado por el cambio climático y luego en una encuesta general este tema no figure o aparezca relegado frente a otros. También se connota en preguntas sobre preocupación ambiental, donde usualmente los entrevistados se valoran

14Disponible en http://awsassets.wwfar.panda.org/downloads/graficos_para_gacetilla.pdf. Último acceso en enero de 2017.

15 Disponible en http://climateandenergy.wwviews.org/results/. Último acceso en enero de 2017.

16 Disponible en http://data.myworld2015.org. Último acceso en enero de 2017.

17 Disponible en http://www.clarin.com/sociedad/educacion-prioridad_0_SyFgEwYwmx.html. Último acceso: marzo de 2017.

18 Disponible en http://www.iprofesional.com/notas/231326-Inflacin-y-corrupcin-al-tope-en-el-rankingde-temas-que-ms-preocupan-a-los-argentinos-. Último acceso: marzo de 2017.

19 Disponible en http://www.latinobarometro.org. Último acceso: enero de 2017. 
a sí mismos como preocupados o muy preocupados por la situación ambiental y a otros actores, como gobiernos y empresas, como poco preocupados. Sin embargo, a la hora de generar acciones concretas, muchas de esas personas que se manifestaron muy preocupados dicen no generar acciones que mejoren la situación ambiental, así como consideran que pueden hacer poco o nada para cambiarla ${ }^{20}$. En la encuesta de World Wide Views se identifica que dos terceras partes de los entrevistados manifiestan que es una responsabilidad global enfrentar el cambio climático y que las acciones deben provenir primero del plano global y luego del local.

Existen otros fenómenos ligados a la percepción, como la hipermetropía ambiental, por la cual la población valora como más positiva la situación de su entorno directo que la de entornos más alejados (Uzzel, 2000). Esto puede notarse en la encuesta administrada por Poliarquía para Fundación Vida Silvestre, donde los entrevistados analizan la situación ambiental global de un modo mucho más grave que la local ${ }^{21}$.

La paulatina y reciente vinculación de la agenda pública ambiental con la agenda política y su relación con la baja demanda climática social también pueden ligarse con la agenda de los medios de comunicación, como cuarto aspecto identificado. Diversas investigaciones procuran demostrar que el cambio climático ha tenido poca acogida en los medios de comunicación, exceptuando momentos específicos y especialmente foráneos, como la celebración de las Conferencias de las Partes de la Convención (Nosty, 2009; González Alcaraz, 2010; Gaviratti, 2012). Sin embargo, la asiduidad de la ocurrencia de fenómenos extremos en territorio nacional que coincidió con la finalización del proceso de negociación internacional del Acuerdo de París, y a su vez, con discursos políticos oficialistas y opositores referidos al tema ${ }^{22}$, junto con un proceso de identificación, formación de intereses e internalización de ideas, derivaron en una mayor cobertura periodística nacional.

Algunas de las principales referencias discursivas al cambio climático se realizaron en el contexto de las inundaciones en la provincia de Buenos Aires en agosto de 2015, en el marco de la campaña presidencial. El entonces gobernador de la provincia y candidato oficialista a la Presidencia de la República, Daniel Scioli, afirmó "nuestro enemigo es el cambio climático". La crítica de la oposición a las palabras de Scioli no se concentró en negar el cambio climático ni sus efectos, sino en afirmar que "el Estado debe invertir y no gastar", y en la necesidad de lograr financiamiento externo a baja tasa de modo de inver-

20 Ver también Cussianovich, 2014.

21 Este fenómeno es reconocido por diversos estudios y encuestas de opinión ambientales en otros países, como el Ecobarómetro Andaluz (Bueno, 2014).

22 Discurso de Presidenta Cristina Fernández en encuentro con empresarios italianos en junio de 2011; Discurso de Presidenta Cristina Fernández en la Sesión Plenaria de la II Cumbre de CELAC en enero de 2014, disponible en http://www.cfkargentina.com/discurso-completo-de-cristina-en-la-cumbre-celac/; Discurso de Presidenta Cristina Fernández en la inauguración de la Central Nuclear Atucha II en febrero de 2015, disponible en https://www.mrecic.gov.ar/palabras-de-la-presidenta-cristina-fernandez-de-kirchneren-la-inauguracion-de-la-central-nuclear; Declaraciones gobernador de Buenos Aires y candidato presidencial por el oficialismo en agosto de 2015 con motivo de las inundaciones en la misma provincia, disponible en https://noticias.terra.com/mundo/culpa-scioli-a-cambio-climatico-por-inundaciones-enargentina,b85f92deeb17c376f3205d1e2cc84611k0h9RCRD.html. Último acceso: enero de 2017. 
tir en infraestructura y evitar los problemas más allá de las inclemencias climáticas ${ }^{23}$.

En quinto lugar, cabe notar el cambio de visión del mundo que impactó en la política exterior argentina (PEA) durante el período analizado. Dicha política asumió un tono crecientemente contestatario con relación a Estados Unidos y a Europa, y cada vez más cercana a otros actores como China o India, en una mirada benévola de la cooperación Sur-Sur.

La Presidenta Cristina Fernández, en ocasión de asumir la Presidencia del G77 más China en el año $2011^{24}$, se refirió a los desbalances que viven los países en desarrollo en comparación con los países desarrollados en el contexto del multilateralismo, especialmente financiero. En tal sentido afirmó:

Los desbalances fuertes también en una situación de aparición de nuevos actores de los cuales China, uno de nuestros integrantes es también un actor, un nuevo actor muy importante a nivel global... El nuevo escenario internacional está protagonizado por países del G-77 más China. Sin embargo, en los sistemas de decisión institucionales, multilaterales nuestros países no tienen representación acorde con su nivel y con su magnitud, en la adopción de decisiones.

El cambio de visión del mundo y sus participantes se asocia a una dinámica en la que actores del Sur, con dilemas similares a la Argentina como la reducción de la pobreza y la búsqueda del desarrollo, eran vistos como "amigos". A su vez estos actores se constituían en principales socios comerciales, lo que se vincula al incremento del precio internacional de los commodities.

Esta condición de la PEA se trasmutó a las negociaciones multilaterales en diversos foros, incluyendo a la CMNUCC, donde la Argentina se volcó al grupo de negociación liderado por China e India, LMDC. Las posiciones del grupo durante las negociaciones previas a París se caracterizaron por un discurso de fuerte crítica a los países desarrollados y su incumplimiento de los compromisos de la CMNUCC, así como la búsqueda de éstos de trasladar sus responsabilidades a los países en desarrollo. De este modo, LMDC y Argentina en particular, se mostraron reticentes a mantener un liderazgo en materia de mitigación y duramente renuentes a adoptar cualquier compromiso de financiamiento climático internacional, que contravendría la obligación de los donantes, según el artículo 4 de la CMNUCC. Esto no sólo se evidenció en las reuniones de la Convención, sino también en otros foros como el G20, así como en la negociación de la Agenda de Acción de Addis Abeba sobre financiamiento para el desarrollo, entre otros.

En sexto lugar, e íntimamente ligado a la faceta internacional de la política pública expresada en el párrafo anterior, la política climática argentina estuvo franqueada por una política energética basada en la quema de fuentes fósiles, bajo la creencia de que las políticas ambientales no se identifican con las políticas de desarrollo. Con lo cual, la posesión y recuperación de recursos naturales identificados como estratégicos y su explotación bajo la justificación del desarrollo no debían verse impedidos por compromisos internacionales de reducción de emisiones que, además, debían ser encabezados por los países desarrollados

23 Discurso del entonces candidato presidencial Mauricio Macri en almuerzo del Consejo Interamericano de Comercio y producción en agosto de 2015, disponible en

http://www.infobae.com/2015/08/13/1748192-las-cinco-promesas-que-les-hizo-macri-los-empresarios/. 
como producto de su responsabilidad histórica en la generación del cambio climático. Cabe notar que esta dinámica de la política ambiental (climática en particular) versus políticas de desarrollo como binomio dicotómico constituye una creencia enraizada en muchos de los líderes políticos, así como en el empresariado, en Argentina.

En el discurso dado por la Presidenta Cristina Fernández ante la Asamblea General de Naciones Unidas en septiembre de 2010 se refirió a la dificultad para lograr un acuerdo climático:

no hemos podido llegar a un acuerdo porque en realidad no se está planteando una solución justa y equitativa en esta materia en cuánto y en quiénes son los que tienen que hacerse cargo, en su gran mayoría, de lo que es el pasivo en materia de contaminación ambiental. No es justo que los países en desarrollo, que a duras penas con su crecimiento económico han podido superar niveles de pobreza y brecha social, sean precisamente los que tengan que hacerse cargo del pasivo ambiental que históricamente es producto de los países desarrollados (...). El establecer también metas y objetivos que sean viables y que sean congruentes con la necesidad de que siga creciendo la economía, obligan a un replanteo serio de lo que son nuestras metas en materia de calentamiento global. ${ }^{25}$

En función de lo afirmado, comprendemos que la construcción social de poder climático radica en la articulación entre los seis aspectos. No se trata de una elite política o incluso un empresariado no interesado en el cambio climático, analizando esto como desvinculado de una sociedad altamente demandante en este tópico. El modelo político sostenido por las administraciones que se extendieron entre 2003 y 2015 respondió a los intereses y creencias de una importante cantidad de argentinos que recurrieron a las urnas en tres oportunidades para refrendar su acción, y además, resultaron muy cercanos a alcanzar un cuarto período electoral en 2015.

Si bien el 91\% de los argentinos (encuesta de World Wide Views) reconoce que el cambio climático no es una prioridad política nacional y otra gran mayoría considera que se deberían tomar medidas aunque otros Estados no lo hagan, la dimensión de la acción propia revela que la disposición a actuar es más baja. Desde nuestra perspectiva, es esta dimensión la que pone de manifiesto la relación entre la baja prioridad de la agenda climática en la agenda política nacional en el período y la escasa demanda social climática (Calvo y Aguado, 1997; Lezama, 2004).

\section{Conclusiones}

El objetivo principal del trabajo fue abordar la cuestión del poder climático de Argentina, en el marco de las negociaciones multilaterales entre 2003 y 2015. Para ello, se comenzó con un análisis de tipo material-racionalista y luego se procedió con uno de orden constructivista. En función de las diferencias metodológicas, epistemológicas y ontológicas de cada enfoque, resultaron dos respuestas de distinta naturaleza. 
El primer modelo presentado, permitió abordar el poder de Argentina en las negociaciones focalizado en su acumulación y en la interacción con la estructura de incentivos y costos potenciales afrontados por el país. En base a esto, se observó que Argentina es un actor que no posee la capacidad de ejercer una influencia de primer orden en las negociaciones y que necesita un acuerdo internacional, dada su débil alternativa a no acordar. Del mismo modo, señalamos que para cumplir dichos objetivos se adoptó una estrategia de tipo defensiva, de confrontación moderada, tal como permite su vulnerabilidad no extrema, y como sugieren su percepción de la negociación como enfocada en ganancias relativas, la defensa de puntos clave relacionados con los intereses de los países centrales, y las características de la coalición doméstica de gobierno.

Por su parte, el segundo enfoque ha buscado abordar la construcción del poder climático social de Argentina focalizada en aspectos identitarios e intereses que explican y significan las condiciones materiales evidenciadas en la primera parte de la contribución. En este punto, hemos señalado seis aspectos de vinculación de las políticas climáticas argentinas ligados a la inclusión del tópico en la agenda política: la relación de dicha agenda con la denominada agenda pública; la demanda social climática; la agenda mediática; el cambio de visión del mundo transmutado a la PEA y a las negociaciones climáticas; y la dicotomía desarrollo/protección ambiental. Si bien la internalización societaria de las causas y consecuencias del cambio climático y por consiguiente, la demanda climática social ha sido baja; ha ido en aumento en el período. Las políticas públicas son el resultado de la confluencia de distintas identidades, intereses y creencias sociales. Este cambio paulatino de un discurso donde el cambio climático estaba ausente, a uno donde figura como justificativo de eventos extremos constituye un primer paso hacia la deconstrucción del de la oposición entre política ambiental y política de desarrollo de la política ambiental como opuesta a la política de desarrollo. A su vez, este cambio paulatino no solo aparece en el discurso político, sino en agenda de los medios de comunicación y favorece en conjunto el proceso de construcción de agenda pública ambiental. Sin embargo, la internalización como antecedente del cambio cultural sigue inmadura.

La elección del enfoque constructivista desde la perspectiva de Alexander Wendt implica reconocer las diferencias ontológicas de los enfoques teóricos a la vez que tender algunos puentes entre dichas tradiciones. Ponemos en evidencia que el análisis materialista impide dos procesos que consideramos significativos en el análisis del poder climático. En primer lugar, el racionalismo dificulta analizar los cambios evidenciados en los intereses e identidades de Argentina lo que interactúa conjuntamente con los aspectos materiales y el contexto internacional, generando distintas motivaciones a involucrarse en mayor o menor medida en las negociaciones climáticas. En segundo lugar, el racionalismo tiende al statuquísmo justamente por su sobrevaloración de la base material. Esto impide una construcción teórica que apunte al cambio social en la medida que el cambio debería asentarse sobre modificaciones ostensibles en la base material, lo cual no ha sucedido en el caso argentino no sólo en el período de estudio sino desde que se adoptó la Convención.

Si bien ambas perspectivas identificaron que la política climática externa durante el período analizado tuvo un carácter defensivo, las conjugaciones de fuerzas y los motivos por los cuales esto se produjo son distintos. En el primer enfoque, los intereses del actor se encuentran caracterizados por ser consecuencias de factores materiales, tales como la medida de su economía, el nivel de las emisiones, la vulnerabilidad ambiental, o las características de su coalición de gobierno, sin una problematización de las ideas que signi- 
fican dichos factores. Esto sucede porque, tal como afirma Wendt (1999), el racionalismo se encuentra metodológicamente incapacitado para explicar cómo se construyen los intereses o las identidades. Más bien, el racionalismo identifica y analiza los incentivos que afectan los comportamientos y en tal esquema los intereses e identidades resultan dados. En este sentido, comprender el proceso de formación de intereses e identidades es significativo para poder construir una teoría social que apunte al cambio y porque para que esto ocurra es necesario comprender las ideas que significan las identidades y capacidades en los procesos sociales.

\section{Bibliografía}

Abbott, Kenneth (2012), "The transnational regime complex for climate change", Environment and Planning C: Government and Policy, vol. 30, nro. 4, pp. 571-590.

Below, Amy (2009), "Climate Change Decisions: International Influences on Argentina's Foreign Policy Decision Making", Ponencia presentada en American Political Science Association Convention, Toronto.

Bially Mattern, Janice (2010), "The concept of power and the (un)discipline of International Relations", en C. Reus-Smit, \& D. Snidal, The Oxford Handbook of International Relations, New York: Oxford University Press, pp. 691-698.

Bouille, Daniel y Osvaldo Girardin (2002), "Learning from the Argentine voluntary commitment", en Kevin Baumert, Odile Blanchard, Silvi Llosa y James Perkaus, Building on the Kyoto Protocol: The options for protecting the Climate, Estocolm: World Resources Institute, pp.135-156.

Bueno, María del Pilar (2010), “Política Exterior y Medio Ambiente en la Argentina. La Influencia de los actores subnacionales y no estatales en la toma de decisiones entre 1989 y 2009", Tesis doctoral, Universidad Nacional de Rosario.

Bueno, María del Pilar (2012), “Los vaivenes de la diplomacia climática argentina (1989-2011)”, en Carla Morasso y Gisela Pereyra Doval, Argentina y Brasil: Proyecciones Internacionales, Cooperación Sur-Sur e Integración, Rosario: UNR Editora, pp.134-154.

Bueno, María del Pilar (2014), "El ecobarómetro, la conciencia ambiental y las propuestas electorales en Andalucía”, Revista Rupturas, Costa Rica, v. 4, no1, pp. 24-49.

Bueno, María del Pilar (2015), Las negociaciones climáticas internacionales y la posición argentina como actor de peso medio, Rosario: Observatorio de Política Exterior Argentina.

Bueno, María del Pilar (2017), "Política Climática Exterior Argentina en el contexto del cambio ambiental global (1990-2015)”, en Griselda Günther y R.A. Gutiérrez, La política del ambiente en América Latina: una aproximación desde el cambio ambiental global, UAM-X-CLACSO.

Calvo, Mariano e Irene Aguado (1997), El medio ambiente en la opinión pública: Tendencias de opinión. Demanda social. Análisis y gestión de la opinión pública en materia de medio ambiente. Comunicación medioambiental en la Administración y en 
la empresa, Madrid: Mundi-Prensa Libros.

Creus, Nicolás (2013), "El concepto de poder en las relaciones internacionales y la necesidad de incorporar nuevos enfoques", Revista Estudios internacionales, vol. 45, no 175, pp. 63-78.

Cussianovich, Ernesto (2014), El medio ambiente en la opinión pública, Informe Ambiental Anual FARN 2014, pp. 277-287.

Franchini, Matías (2011), "Sem lugar no mundo: A Argentina na politica internacional das mudanças climáticas", Tesis de maestría, Universidade de Brasília.

Gavirati, Pablo (2012), "Periodismo local y cambio climático global análisis discursivo de la COP-15 en la prensa argentina", Razón y Palabra, México, v. 17, n 079.

González Alcaraz, Luis (2010), “Este asunto no es nuestro - El cambio climático en la prensa escrita de referencia en Argentina", Kairos, San Luis, año 14, no 26.

Gutiérrez, Ricardo y Fernando Isuani (2014), "The emergence of state and social environmentalism in Argentina", Revista de Administração Pública, v. 48, Rio de Janeiro, no 2, pp. 295-332.

Guzzini, Stefano (2005), "The concept of power: a constructivist analysis", Millenium: Journal of International Studies, Londres, v. 33, no 3, pp. 495-521.

Haas, Peter (2004), "When does power listen to truth? A constructivist approach to the policy process", Journal of European public policy, vol. 11, nro. 4, pp. 569-592.

Hill, Christopher (2003), The changing Politics of Foreign Policy, New York: Palgrave and MacMillan.

Hurd, Ian (2008), “Constructivism”, en Christian Reus-Smit y Duncan Snidal, The Oxford Handbook of International Relations, Oxford: Oxford University Press, pp. 298-316.

Keohane, Robert; Victor, David (2011), "The regime complex for climate change", Perspectives on politics, vol. 9, nro. 1, pp. 7-23.

Keohane, Robert (1993), Instituciones Internacionales y Poder Estatal, Buenos Aires: GEL.

Keohane, Robert y Joseph Nye (1988), Poder e Interdependencia: la política mundial en transición, Buenos Aires: GEL.

Krasner, Stephen (1989), Conflicto estructural: el Tercer Mundo contra el Liberalismo Global, Buenos Aires: GEL.

Kreft, Sönke, David Eckstein e Inga Melchior (2017), Global Climate Risk Index 2017, Bonn: German Watch.

Levitsky, Steven y Kenneth Roberts (2011), The Resurgence of the Latin American Left, Baltimore: The Johns Hopkins University Press.

Lezama, José Luis (2004), La construcción social y política del medio ambiente, México: El Colegio de México AC.

McKibben, Heather Elko (2015), State Strategies in International Bargaining: Play by the Rules or Change Them?, Cambridge: Cambridge University Press. 
Mearsheimer, John (2001), The Tragedy of Great Power Politics, New York: W. W. Norton \& Company.

Merke, Federico y Diego Reynoso (2016), “Dimensiones de política exterior en América Latina según juicio de expertos", Estudios Internacionales, Santiago de Chile, no 185, pp.107-131.

Moravcsik, Andrew (2008), "The New Liberalism", en Christian Reus-Smit y Duncan Snidal, The Oxford Handbook of International Relations, Oxford: Oxford University Press, pp. 234-254.

Navarrete, David (2010), “Climate Change and power: Isn't it all about politics?", Environment, Politics and Development Working Paper Series, Londres, Paper 32.

Nosty, Bernardo Díaz (2009), “Cambio climático, consenso científico y construcción mediática. Los paradigmas de la comunicación para la sostenibilidad", Revista Latina de comunicación social, Tenerife, no 64, pp. 99-119.

Paterson, Matthew (2003), Global warming and global politics, New York: Routledge.

Pettenger, Mary (2007), "The Social Construction of Climate Change: Power, Knowledge, Norms, Discourses", Routledge.

Picó Garcés, María Josep (2013), “La crisis económica versus el cambio climático”, Actas de las Jornadas Internacionales Medios de Comunicación y Cambio Climático, Càtedra de Divulgació de la Ciència, UCC+i, Universitat de València, pp. 177187.

Rong, Fang (2010), “Understanding developing country stances on post-2012 climate change negotiations: Comparative analysis of Brazil, China, India, Mexico, and South Africa", Energy Policy, no 38, pp. 4582-4591.

Rosa, Eugene; Dietz, Thomas (1998), “Climate change and society: Speculation, construction and scientific investigation", International Sociology, vol. 13, nro. 4, pp. 421455.

Rubio Ferreres, José María (2009), “Opinión pública y medios de comunicación. Teoría de la agenda setting", Gazeta de Antropología, Granada, v. 25, no 1, .

Secretaría de Ambiente y Desarrollo Sustentable (2015), Tercera Comunicación Nacional de la República Argentina a la Convención Marco de las Naciones Unidas sobre Cambio Climático, Buenos Aires: Jefatura de Gabinete de Ministros.

Solingen, Etel (1998), Regional Orders at Century's Dawn: Global and Domestic Influences on Grand Strategy, New Jersey: Princeton University Press.

Solingen, Etel (2001), "Mapping Internationalization: Domestic and Regional Impacts", International Studies Quarterly, Oxford, v. 45, no 4, pp. 517-555.

Uzzel, David (2000), "The psyco-spatial dimension of global environmental problems", Journal of environmental psychology, v. 20, n , pp. 307-318.

Viola, Eduardo, Matías Franchini y Thais Ribeiro (2012), “Climate governance in an international system under conservative hegemony: the role of majors powers", Revista Brasileira de Política Internacional, Brasilia, v. 55, Esp., pp. 9-29. 
Waltz, Kenneth (1988), Teoría de la Política Internacional, Buenos Aires: GEL.

Wendt, Alexander (1999), Social theory of international politics, Cambridge: Cambridge University Press.

Wendt, Alexander (1992), "Anarchy is what states make of it: the social construction of power politics", International Organization, Cambridge, v. 46, № 2, pp. 391-425.

Yamin, Farhana; Depledge, Joanna (2004). The international climate change regime: a guide to rules, institutions and procedures, Cambridge University Press. 